\title{
Multi-objective optimization of quality in laser cutting based on response surface model
}

\author{
Hao Huijuan , Wang Maoli ,Hao fengqi \\ Shandong Provincial Key Laboratory of Computer Network \\ Shandong Computer Science Center \\ Jinan, China \\ haohj@keylab.net
}

\begin{abstract}
Prediction and optimization of quality characteristics is an important means to improve the quality of laser cutting. Kerf width (KW) and material removal rate(MRR) are selected as the quality characteristics in this paper. The fitting response surface models (RSM) of KW and MRR are considered as the optimization objective function in pulsed Nd: YAG laser cutting of alloy steel for multi-objective optimization. An improved Pareto genetic algorithm is used in the optimization, and the significant factors have been found. The predicted results are basically consistent with the experimental. Therefore, the method used in this paper can be used for optimization of KW and MRR in pulse Nd: YAG laser cutting. The study can provide theoretical basis for the prediction and optimization of quality in laser cutting.

Keywords-laser cutting; multi-objective optimization; RSM;
\end{abstract} improved Pareto genetic algorithm

\section{INTRODUCTION}

Since its introduction, laser cutting has always been a major area of research for improving the quality of cutting. In order to analyze and improve different quality characteristics, many researchers conducted theoretical and experimental study[1,2]. In most experimental studies, the researchers change one processing parameter at one time to analyze its impact on the quality characteristics. So, the study requires a lot of experiments. To overcome this problem, some researchers began the study to change different input parameters simultaneously. For this purpose, the design of experiment(DOE), such as factorial design, Taguchi method and response surface method, is applied to the parameter optimization and modeling[3-5]. The study based on DOE is mainly focused on the optimization of single quality characteristic[6].The experiment results show that the optimization of single characteristic parameter may cause the deterioration of other quality characteristics. In the last few years, there has been growing interest in the use of pulsed Nd:YAG lasers for precision cutting of thin sheetmetals and for these applications that demands narrow kerf widths, small HAZ and intricate cut profiles[7,8]. Therefore, the studies in this paper concentrate on multi-objective optimization of pulse Nd: YAG laser cutting.

The relationship between input and output parameters in laser cutting process is non-linear and complicated. The conventional methods of modeling and optimization require large amount of experiments. To overcome these difficulties, the method of modeling and optimization based on artificial intelligence, such as artificial neural network, fuzzy logic and genetic algorithm are used[9-11]. Pareto genetic algorithm is an optimization method based on the extension of conventional genetic algorithm, and has a very good advantage on multi-objective optimization. The Pareto genetic algorithm is used in this paper to conduct the simultaneous of $\mathrm{KW}$ and MRR, and the set filter is improved. When the relationship between the response and factors is non-linear, the response surface method becomes the best tool for optimization of process parameters. In this paper, the fitting second-order response surface models are used as the objective function of $\mathrm{KW}$ and MRR, and the improved Pareto genetic algorithm is used to predict KW and MRR. The optimization process takes fully advantage of the genetic algorithm and RSM model in the disposing of non-linear problem.

\section{RSM MODEL}

Response surface method gets the second order mathematical relation model as a real function approximation by fitting the response output and influence factors. The method carries on the analysis and optimization, and it defines a set of factors and levels to achieve optimal response variables. The second-order response surface model by least squares fitting is shown as expression 2.1.

$$
\hat{y}_{j}=\hat{\beta}_{0}+\sum_{i=1}^{k} \hat{\beta}_{i} x_{i}+\sum_{i=1}^{k} \hat{\beta}_{i i} x_{i}^{2}+\sum_{i<j}^{k} \hat{\beta}_{i j} x_{i} x_{j}
$$

Where all $\hat{\beta}^{\prime} s$ are the regression coefficients, determined numeri-cally by using leasts quare fit method, $X_{i}$ are the different control factors changing from $i=1$ to $\mathrm{k}$, $\mathrm{k}$ is the total number of control factors, $\hat{y}_{j}$ are the different responses ( $\mathrm{j}$ are the numbers of response used).In this study $j=2, i=4$, has been used.

\section{A. Models of KW and MRR}

The final models for KW and MRR are developed from the experimental data obtained from CCRD experimental matrix. The coefficients for second order regression models are determined with the help of Matlab 7.0. The final models obtained are as follows. 
$K W=0.2841+0.0007 x_{1}+0.3620 x_{2}-0.0127 x_{3}$

$-0.0008 x_{4}+0.0028 x_{1}^{2}-0.0292 x_{2}^{2}+0.0005 x_{3}^{2}$

$-0.0066 x_{1} x_{2}-0.0001 x_{1} x_{4}-0.0092 x_{2} x_{3}$

$+0.0001 x_{2} x_{4}$

$M R R=-10.3643-11.5384 x_{1}-121.4368 x_{2}$

$+5.0106 x_{3}+1.8571 x_{4}+3.4007 x_{1}^{2}$

$-40.4445 x_{2}{ }^{2}+0.1679 x_{3}{ }^{2}-0.0004 x_{4}{ }^{2}$

$+45.7952 x_{1} x_{2}-2.8905 x_{1} x_{3}+0.1182 x_{1} x_{4}$

$+2.2334 x_{2} x_{3}+0.1857 x_{2} x_{4}-0.0452 x_{3} x_{4}$

Where $x_{1}$ is gas pressure $\left(\mathrm{kg} / \mathrm{cm}^{2}\right), \quad x_{2}$ is pulse width(Ms), $x_{3}$ is pulse frequency $(\mathrm{Hz})$, and $x_{4}$ is cutting speed(mm/min). In order to test the accuracy of the fitted models, F-values, P-values and coefficients of KW and MRR are calculated. The results are shown in Table I .

TABLE I. RESULTS OF ANOVA FOR DEVELOPED MODELS

\begin{tabular}{|c|c|c|}
\hline Parameter & KW & MRR \\
\hline Correlation coefficient $\mathbf{r}^{\mathbf{2}}$ & 0.9297 & 0.9684 \\
\hline F-value & 14.1593 & 20.6165 \\
\hline P-value & 0.0000 & 0.0000 \\
\hline Residual sum of squares & 0.0001 & 69.3498 \\
\hline
\end{tabular}

From Table 1, it can be seen that the coefficient of KW and MRR is 0.9297 and 0.9684 respectively. F-value is 14.1593 and 20.6165 respectively. F-values and P-values are both within the allowed range. P-values are less than 0.0001 . All shows that the fitting for KW and MRR is good, and the developed regression models are appropriate.

\section{B. Parameters analysis}

As can be seen from expression 2.2 that $x_{2}, x_{3}$, square effect of $x_{1}$,square effect of $x_{2}$, and interaction effect of $x_{2}$ and $x_{3}$, interaction effect of $x_{1}$ and $x_{2}$ are significant factors of KW. The coefficients of these parameters are higher than others. Similarly, $x_{1}, x_{2}$, square effect of $x_{2}$, interaction effect of $x_{1}$ and $x_{2}$ are significant factors of MRR.

\section{PREDICTION OF KW AND MRR}

\section{A. Modelling}

$\mathrm{KW}$, cutting surface roughness, heat affected zone, solidified layer, adhesion debris, MRR, etc. are used to characterize the quality of laser cutting. KW and MRR are selected in this paper. According to the experimental analysis, $\mathrm{KW}$ is the smaller the better, while MRR is the bigger the better. So the model of the quality optimization can be described by the following mathematical formula.

$$
\left\{\begin{array}{l}
\min K W=f_{1}\left(x_{1}, x_{2}, x_{3}, x_{4}\right) \\
\max M R R=f_{2}\left(x_{1}, x_{2}, x_{3}, x_{4}\right)
\end{array}\right.
$$

Where, $x_{1}$ is gas pressure, $x_{2}$ is pulse width, $x_{3}$ is pulse frequency, $x_{4}$ is cutting speed.
The formula 2.2 and 2.3 are selected as the objective function of KW and MRR in this paper.

\section{B. Prediction based on the improved Pareto genetic algorithm}

Pareto genetic algorithm [12] has a very good advantage on multi-objective optimization. Because it is multi-objective optimization, the options for practical Pareto solutions are many, the filter volume of Pareto solution set is particularly important. If it is set too large, the computation amount will be too large, and the search speed of Pareto algorithm will be influenced; if be set too small, the good solution will be missed, and the accuracy will be affected. To balance the precision and the speed, the improved Pareto solution filter used in reference [13] is adopted, and the simultaneous optimization of KW and MRR is conducted in this paper.

The control parameters used in Pareto genetic algorithm are shown as follows. The population size is 60 ; the max generation is 100; hybrid probability is 0.8 ; mutation rate is 0.1 ; the Pareto solution set filter size is $40 ; d_{\text {pre }}$ is set to be $5 ; \beta$ is $15^{0}$.

\section{RESULTS AND ANALYSIS}

In order to verify the accuracy of the above algorithm in the actual laser cutting, KW and MRR are predicted in pulse Nd:YAG laser cutting (200W), and compared with the experiment results.

\section{A. Processing parameters}

Auxiliary gases: oxygen; focal length:50.0mm;nozzle diameter:1.0mm; nozzle distance:1.0mm; thickness of Aluminum alloy steel material: $0.5 \mathrm{~mm}$. The specific parameters are shown in Table II.

\section{TABLE II. CUTTING PARAMETERS}

\begin{tabular}{|c|c|c|}
\hline Cutting parameters & level & values \\
\hline Gas pressure & $\mathrm{kg} / \mathrm{cm}^{2}$ & $1.5-4.0$ \\
\hline Pulse width & $\mathrm{Ms}$ & $1.0-1.4$ \\
\hline Pulse frequency & $\mathrm{Hz}$ & $20-28$ \\
\hline Cutting speed & $\mathrm{mm} / \mathrm{min}$ & $25-75$ \\
\hline
\end{tabular}

\section{B. Comparison with experiment results}

The comparison of the predicted results with the experimental results is shown in Figure 1. It can be seen that the predicted results agree with the experimental results. The developed models in this paper can be used as the models of KW and MRR. 

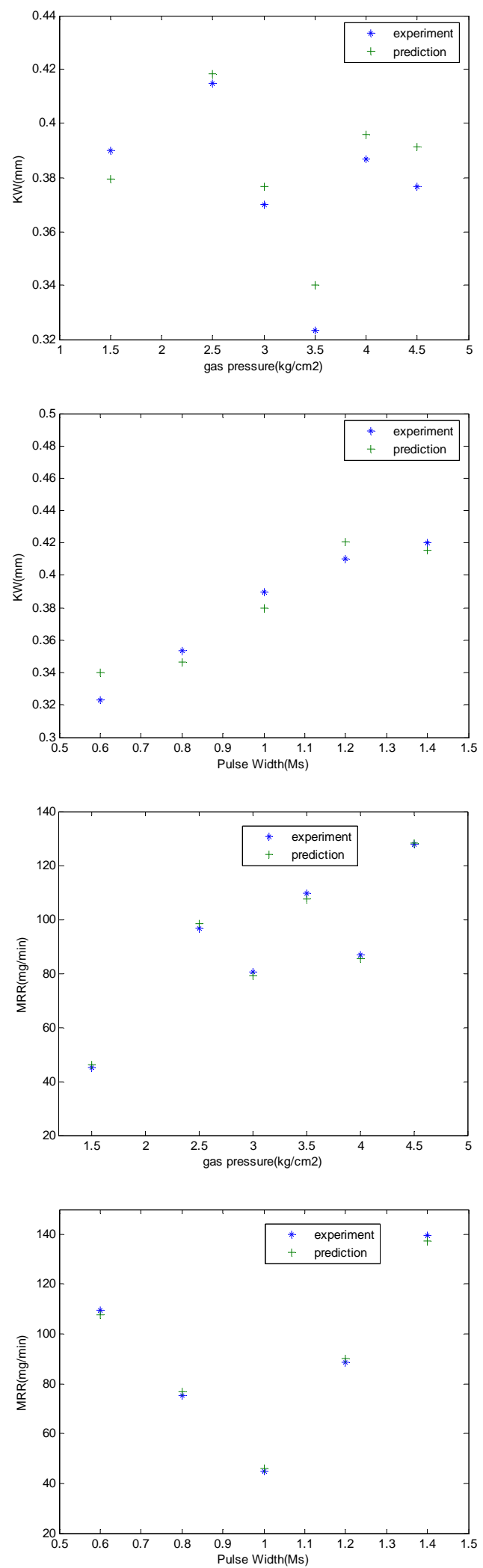

Figure 1. comparison with the experimental results

\section{Comparison with unimproved algorithm}

The comparison of the improved Pareto genetic algorithm with the traditional algorithm is shown in figure 2 . As can be seen, the improved Pareto genetic algorithm has certain advantage; its frontier of the solution set is more uniform.

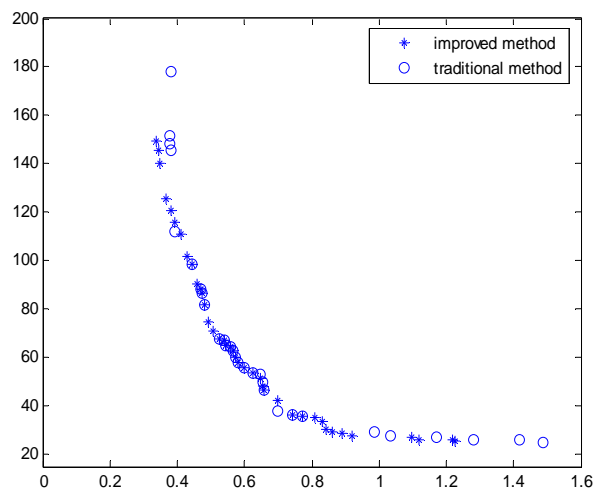

Figure 2. Comparison of the improved method with traditional

\section{Optimization results}

Since in the Pareto front, none of the solution may be said absolutely better than the other solutions. All sets of solutions in the Pareto front are optimal solutions depending upon the requirement of the actual processing. The aim of this paper is to improve both of the quality characteristics (KM and MRR) simultaneously.Since the optimum levels of control factors will be that levels for which both of the quality characteristics are showing better values. Considering equal importance of both the KW and MRR, The values of KW and MRR corresponding to the optimum level of control factors have been found as 0.3412 and 164.1342. The correspond processing parameters are $x_{1}=3.5 \mathrm{~kg} / \mathrm{cm}^{2}, x_{2}=0.6 \mathrm{Ms} \quad, \quad x_{3}=28 \mathrm{~Hz}$, $x 4=100 \mathrm{~mm} / \mathrm{min}$.

\section{CONCLUSIONS}

The models of KW and MRR are developed in this paper. The F-values of $\mathrm{KW}$ and MRR are both in the permitted range. Also, P-values are suitable. So, the developed models are adequate for $\mathrm{KW}$ and MRR, and selected as the objective function of the improved Pareto genetic algorithm. Compared with the traditional Pareto genetic algorithm, the solution set frontier of the improved method is more uniform. The prediction results are basically consistent with the experiments. It can be seen that the developed models can be used as the models of $\mathrm{KW}$ and MRR, and the improved Pareto genetic algorithm can be used to predict and optimize for $\mathrm{KW}$ and MRR in Nd:YAG laser cutting.

\section{ACKNOWLEDGMENT}


Supported by Shangdong Province Young and MiddleAged Scientists Research Awards (No. BS2010DX029) and (No. ZR2010FQ018).

\section{REFERENCES}

[1] Shanjin LV, Yang Wang. An investigation of pulsed laser cutting of titanium alloy sheet. Opt Lasers Eng 2006;44:1067-77.

[2] Tsai Chuwan-Huei, Chen Hong-Wen. Laser cutting of thick ceramic substrates by controlled fracture technique. J Mater Process Technol 2003;136:166-73.

[3] Yilbas BS,Rashid M.CO2 laser cutting of incoloy $800 \mathrm{HT}$ alloy and its quality assessment. Lasers Eng 2002;12:135-45.

[4] Almeida IA, de Rossi W, Lima MSF, Berretta JR, Ngueira GEC, Wetter NU, et al. Optimization of titanium cutting by factorial analysis of pulsed Nd:YAG laser parameters. J Mater Process Technol 2006;179:105-10.

[5] Yilbas BS,Hyder SJ,Sunar M.The Taguchi method for determining CO2 laser cut quality.J LaserAppl 1998;10(2):71-7.

[6] Dubey AK, Yadava V. Robust parameter design and multi-objective optimiza- tion of laser beam cutting for aluminium alloy sheet. Int $J$ Adv Manuf Technol 2008;38:268-77.
[7] Ghany AK, Newishy M. Cutting of $1.2 \mathrm{~mm}$ thick austenitic stainless steel sheet using pulsed and CW Nd:YAG laser. Journal of Material Processing Technology 2005;168:438-47.

[8] Thawari G, Sarin Sundar JK, Sundararajan G, Joshi SV. Influence of process parameters during pulsed ND:YAG laser cutting of nickelbase superalloys. Journal of Materials Processing Technology 2005;170:229-39.

[9] Pradhan MK, Biswas CK. Neuro-fuzzy model and regression model a compar- ison study of MRR in electrical discharge machining of D2 tool steel. Int J Math Phys Eng Sci 2009;3:48-53.

[10] Sivarao, Brevern P, Tayeb NSM, Vengkatesh VC. Modeling, testing and experimental validation of laser machining micro quality response by artificial neural network. Int J Eng Technol 2009;09:1616.

[11] Syn CZ, Mokhtar M, Feng CJ, Manurung HPYupiter. Approach to prediction of laser cutting quality by employing fuzzy expert system. Expert Syst Appl 2011;38:7558-68.

[12] WANG Xiao-peng. Pareto genetic algorithm for multi-objective optimization design. Systems Engineering and Electronics. Systems Engineering and Electronics 25(12) (2003), 1558-1561.

[13] H-J. Hao, J-Y. Xu,J. Li. Prediction of Laser Cutting Quality Based on Improved Pareto Genetic Algorithm. Lasers in Eng., 0:1-14. 